\title{
ON THE FRAME FIXING IN QUANTUM GRAVITY
}

\author{
S. MERCURI \\ ICRA - International Center for Relativistic Astrophysics \\ G. MONTANI \\ ICRA - International Center for Relativistic Astrophysics \\ Dipartimento di Fisica, Università di Roma "La Sapienza", Piazzale Aldo Moro 5, \\ I-00185, Roma, Italy
}

\begin{abstract}
We provide a discussion about the necessity to fix the reference frame before quantizing the gravitational field. Our presentation is based on stressing how the $3+1$-slicing of the space time becomes an ambiguous procedure as referred to a quantum 4-metric.
\end{abstract}

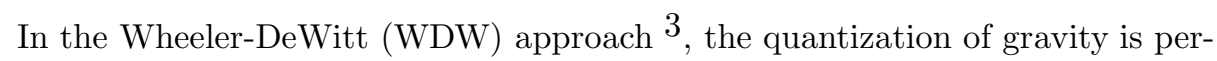
formed in the canonical way, starting from the Arnowitt-Deser-Misner (ADM) action. The use of the ADM formalism 1 is justified by the necessity to obtain Hamiltonian constraints, but the straightforward quantization of such $(3+1)$-picture contains some relevant ambiguities. In fact, the aim of the WDW approach is to quantize the gravitational field in a particular representation and its outcoming provides essentially information on the quantum dynamics of the 3-metric tensor defined on spatial hypersurfaces.

To use the ADM splitting is equivalent to a kind of "gauge fixing", because it is preserved only under restricted coordinates transformations (time displacements and 3 -diffeomorphisms); the point here is that the "gauge fixing" depends on the field we are quantizing and therefore the canonical approach seems to be an ambiguous procedure.

Since in the ADM action the conjugate momenta, $\pi$ and $\pi^{i}$, respectively to the lapse function $N$ and to the shift vector $N^{i}$ are constrained to vanish, then, on a quantum level, the wave functional of the system does not depend on the lapse function and on the shift vector. The ambiguity relies on regarding as equivalent the fully covariant approach and the "gauge fixed" ADM one, in fact passing from $g_{\mu \nu}$ to ADM variables involves a metric dependent procedure, in the sense that we must be able to define a unit time-like normal field $n^{\mu}\left(g_{\mu \nu} n^{\mu} n^{\nu}=-1\right)$, which ensures the space-like nature of $h_{i j}$ (in this respect we recall that $h_{i j} \equiv g_{\mu \nu} \partial_{i} y^{\mu} \partial_{j} y^{\nu}$ corresponds to the spatial components of the 4 -tensor $\left.h_{\mu \nu}=g_{\mu \nu}+n_{\mu} n_{\nu}\right)$. Now the following question arises: how is it possible to speak of a unit time-like normal field 
for a quantum space-time? Indeed such a notion can be recognized, in quantum regime, at most in the sense of expectation values; therefore assuming the existence of $n^{\mu}$ before quantizing the system dynamics makes the WDW approach physically ill defined.

Our point of view is that the canonical quantization of the gravitational field can be performed in a $(3+1)$-picture only if we add, to such a scheme, some information about the existence of the time-like normal field, as shown in 715 , this result can be achieved by including in the dynamics the kinematical action 4, already adopted to quantize "matter" fields on a fixed background 4 . The physical interpretation of such new term either on a classical as well as on a quantum level leads to recognize the existence of a reference fluid and in this sense the analysis of $7|5| 6 \mid$ converges with the literature on the frame fixing problem (see 2 and references therein). We observe that to include the kinematical action can be regarded as a consequence of fixing in the gravity action the lapse function and the shift vector and, therefore, to choose four independent components of the gravitational field, which is just the outcoming of the frame fixing.

A more physical manner to ensure the existence of a time-like vector consists of filling the space time with a fluid which plays the role of real reference frame. Here we discuss on a phenomenological ground, the canonical quantization of the gravitational field plus a dust reference fluid, outlining some relevant differences between the classical and quantum behavior of this system.

The Einstein equations and the conservation law, for the coupled gravity-fluid system, take the form

$$
G_{\mu \nu}=\chi \varepsilon u_{\mu} u_{\nu}, \quad u^{\nu} \nabla_{\nu} u^{\mu}=0, \quad \nabla_{\nu}\left(\varepsilon u^{\nu}\right)=0,
$$

where $G_{\mu \nu}$ and $\chi$ denotes respectively the Einstein tensor and constant.

Remembering a well-known result, it is easy to show that the following relations take place 8

$$
G_{\mu \nu} u^{\mu} u^{\nu}=-\frac{H\left(h_{i j}, p^{i j}\right)}{2 \sqrt{h}}=\chi \varepsilon, \quad G_{\mu \nu} u^{\mu} h_{i}^{\nu}=\frac{H_{i}\left(h_{i j}, p^{i j}\right)}{2 \sqrt{h}}=0 .
$$

Here $h_{i j}(i j=1,2,3)$ denotes the 3 -metric of the spatial hypersurfaces orthogonal to $u^{\mu}$ and $p^{i j}$ its conjugate momenta, while $H$ and $H_{i}$ refer respectively to the super-Hamiltonian and to the super-momentum of the gravitational field. The above relations hold if we make reasonable assumption that the conjugate momentum $p^{i j}$ is not affected by the matter variables (i.e. the fluid term in ADM formalism should not contain the time derivative of the 3-metric tensor). Only the Hamiltonian constraints are relevant for the quantization procedure and, in the comoving frame, when the 4-velocity becomes $u^{\mu}=\{1,0\}\left(N=1 N^{i}=0\right)$, we have to retain also the conservation law $\varepsilon \sqrt{h}=-\omega\left(x^{i}\right) / 2 \chi$, where $h \equiv \operatorname{deth}_{i j}$ and $x^{i}$ denote the spatial coordinates of the comoving frame. Indeed, a crucial point in the above considerations relies on the synchronous nature of the comoving frame as consequence of the geodesic motion of the dust fluid. 
Thus, when the coordinates system becomes a real physical frame, the Hamiltonian constraints read

$$
H=\omega\left(x^{i}\right) \quad H_{i}=0 .
$$

Now, to assign a Cauchy problem for such a system, for which equations (31) play the role of constraints on the Cauchy data, corresponds to provide on a (non-singular) space-like hypersurface, say $\Sigma^{(0)}$, the values $\left\{h_{i j}^{(0)}, p^{(0) i j}, \varepsilon^{(0)}\right\}$; from these values $\omega^{(0)}$ can be calculated by (3).

It follows that, by specifying a suitable initial condition, the value of $\omega^{(0)}$ can be made arbitrarily small; from the constraints point of view, a very small value of $\omega^{(0)}$ means, if $h^{(0)}$ is not so, that the fluid becomes a test one (being $\omega$ a constant of the motion); we emphasize that for finite values of $\omega, h$ should not vanish to avoid unphysical diverging energy density of the fluid.

The canonical quantization of this system is achieved as soon as we implement the canonical variables into quantum operators and annihilate the state functional $\Psi$ via the Hamiltonian operator constraints. Thus the quantum dynamics obeys the following eigenvalue problem:

$$
\widehat{H} \Psi\left(\left\{h_{i j}\right\}, \omega\right)=\omega \Psi\left(\left\{h_{i j}\right\}, \omega\right),
$$

where $\left\{h_{i j}\right\}$ refers to a whole class of 3 -geometries, so that the super-momentum constraint holds automatically.

We stress how the above result is equivalent to the eigenvalues problem obtained in 7 . In the above equation (4), the spatial function $\omega$ plays the role of the superHamiltonian eigenvalue; in this respect, we observe how its values can no longer be assigned by the initial values, but they have to be determined via the spectrum of $\widehat{H}$. We conclude that, in the quantum regime, a real dust reference fluid never approaches a test system.

Moreover the presence of non zero eigenvalues for the super-Hamiltonian removes the so called "frozen formalism" of the WDW equation and confirms the idea that introducing a physical unit time like vector provides a consistent and evolutive canonical quantum gravity dynamics.

\section{References}

1. R. Arnowitt, S. Deser, C. Misner, (1959), Phys. Rev. 116, 1322.

2. J. Bicak, K. Kuchař, (1997), Phys. Rev. D56, 4878.

3. B.S. DeWitt, (1967), Phys. Rev. 160, 1113.

4. K. Kuchař, Canonical Methods of Quantization, (1981), 'Quantum Gravity 2: A Second Oxford Symposium', Clarendon Press, Oxford, pp.329-374.

5. S. Mercuri, G. Montani, (2003), to appear on Int. Jour. Mod. Phys. D, available on gr-qc/0310077.

6. S. Mercuri, G. Montani, (2003), submitted to Class. Quant. Grav., available on grqc/0312077.

7. G. Montani, (2002), Nucl. Phys. B634, 370.

8. T. Thiemann, (2001), available gr-qc/0110034. 